\title{
DIFFERENTIABLE PINCHING THEOREMS FOR SUBMANIFOLDS VIA RICCI FLOW
}

\author{
Hongwei Xu, Fei Huang and Entao Zhao
}

(Received May 1, 2014, revised June 18, 2014)

\begin{abstract}
Two differentiable pinching theorems are verified via the Ricci flow and stable currents. We first prove a differentiable sphere theorem for positively pinched submanifolds in a space form. Moreover, we obtain a differentiable sphere theorem for submanifolds in the sphere $\mathbb{S}^{n+p}$ under extrinsic restriction.
\end{abstract}

1. Introduction. The sphere theorem is an important topic in the study of curvature and topology of Riemannian manifolds. Recently, S. Brendle and R. Schoen [6] proved a remarkable differentiable sphere theorem for Riemannian manifolds whose sectional curvatures lie in the interval $(1 / 4,1]$ by developing the theory and techniques of Ricci flow introduced by R. Hamilton [9]. This improves the topological sphere theorem for quarter-pinched Riemannian manifolds, which was firstly taken up by H. E. Rauch [16] in 1951, and solved by M. Berger [2] and W. Klingenberg [10] around 1960. In 1977, K. Grove and K. Shiohama [8] proved a topological sphere theorem for complete and connected Riemannian manifolds whose sectional curvature $K$ and diameter $d(M)$ satisfy $K \geq 1$ and $d(M)>\pi / 2$. Some other sphere theorems for Riemannian manifolds have also been obtained, see [1, 14, 15, 17, 25], etc.

In 1973, H. B. Lawson and J. Simons [11] proved a topological sphere theorem for closed submanifolds in a unit sphere by using the nonexistence for stable currents on compact submanifolds in a sphere. Let $\mathbb{F}^{n+p}(c)$ denote the complete simply connected space form with constant sectional curvature $c$. K. Shiohama and H. W. Xu [18] improved Lawson-Simons' result and proved a topological sphere theorem for complete submanifolds in $\mathbb{F}^{n+p}(c)$ with $c \geq 0$. Recently, H. W. Xu and J. R. Gu [20] obtained an optimal differentiable sphere theorem for complete submanifolds with pinched scalar curvature in $\mathbb{F}^{n+p}(c)$ with $c \geq 0$. Let $K_{M}$ denote the sectional curvature of a Riemannian manifold $M$. Under the pinching condition of sectional curvature, H. W. Xu and G. X. Yang [23] proved a topological sphere theorem for compact submanifolds in the spherical space form.

2010 Mathematics Subject Classification. Primary 53C20; Secondary 53C40.

Key words and phrases. Submanifolds, differentiable sphere theorem, Ricci flow, stable current, curvature pinching.

Research supported by the National Natural Science Foundation of China, Grant Nos. 11371315, 11201416, and the Trans-Century Training Programme Foundation for Talents by the Ministry of Education of China. 
THEOREM 1.1. Let $M$ be a compact, oriented $n(\geq 4)$-dimensional submanifold in the space form $\mathbb{F}^{n+p}(c)$ with $c>0$. If the sectional curvature of $M$ satisfies

$$
K_{M}>\frac{c}{2}+\frac{n}{8} H^{2}
$$

then $M$ is homeomorphic to the standard $n$-sphere $\mathbb{S}^{n}$.

Let $h$ be the second fundamental form of a compact submanifold in a Riemannian manifold. In 1986, H. Gauchman [7] proved that if $M$ is an $n$-dimensional closed minimal submanifold in $\mathbb{S}^{n+d}$, and if $\|h(u, u)\|^{2}<\frac{1}{3}$ for any unit vector $u \in U M$, where $U M$ is the unit tangent bundle over $M$, then $M$ is a totally geodesic sphere. H. W. Xu, W. Fang and F. Xiang [21] generalized this rigidity result to the case where $M$ is an $n$-dimensional closed submanifold with parallel mean curvature in $\mathbb{S}^{n+d}$. On the other hand, P. F. Leung [12] proved that if $\|h(u, u)\|^{2}<\frac{1}{3}$ holds for any unit tangent vector $u$ at any point on the submanifold, then it is a homotopy sphere. The result was improved to be a topological sphere theorem for complete submanifolds in a sphere by H. W. Xu and W. Fang [19], and further improved to be a differentiable sphere theorem by H. W. Xu and E. T. Zhao [24].

THEOREM 1.2. Let $M$ be an n-dimensional complete submanifold in the unit sphere $\mathbb{S}^{n+p}$. If

$$
\|h(u, u)\|^{2}<\frac{1}{3}, \quad \text { for all } u \in U M,
$$

then $M$ is diffeomorphic to the standard $n$-sphere $\mathbb{S}^{n}$.

Let $h$ be the trace free second fundamental form of a submanifold in a Riemannian manifold. In [22], H. W. Xu, F. Huang and F. Xiang investigated the pinching theorem of $h$ for the submanifolds with parallel mean curvature in a sphere, which extends Gauchman's theorem in [7].

In this paper, using the convergence result of Ricci flow by S. Brendle [5], we obtain several differentiable sphere theorems for complete submanifolds which generalize Theorems 1.1 and 1.2. We first prove the following

THEOREM 1.3. Let $M$ be an $n(\geq 4)$-dimensional oriented complete submanifold in $\mathbb{F}^{n+p}(c)$ with $c>0$. Assume that the sectional curvature of $M$ satisfies

$$
K_{M}>\frac{(n-2) c}{n}+\frac{n H^{2}}{8},
$$

then $M$ is diffeomorphic to the standard $n$-sphere $\mathbb{S}^{n}$.

Next, we investigate the differentiable pinching problem of $h$ and prove the following

THEOREM 1.4. Let $M$ be an n-dimensional complete submanifold in the unit sphere $\mathbb{S}^{n+p}$. If

$$
\|\stackrel{\circ}{h}(u, u)\|^{2}<\frac{1}{3}, \quad \text { for all } u \in U M,
$$

then $M$ is diffeomorphic to the standard $n$-sphere $\mathbb{S}^{n}$. 
In fact, Theorem 1.4 is a consequence of the following theorem and Lemma 3.2 in Section 3.

THEOREM 1.5. Let $M$ be an $n$-dimensional complete submanifold in an $(n+p)$ dimensional point-wise $\delta(>1 / 4)$-pinched Riemannian manifold $N^{n+p}$. Set $\bar{K}_{\max }(x):=$ $\max _{\pi \subset T_{x} N} \bar{K}(x, \pi)$, where $\bar{K}(x, \pi)$ is the sectional curvature of $N^{n+p}$ for $x \in N$ and 2plane $\pi \in T_{x} N$. If

$$
\|\stackrel{\circ}{h}(u, u)\|^{2}(x)<\frac{4}{9} \bar{K}_{\max }(x)\left(\delta-\frac{1}{4}\right), \quad \text { for all } u \in U_{x} M, \quad x \in M,
$$

where $\inf _{x \in N} \bar{K}_{\max }(x)>0$, then $M$ is diffeomorphic to a spherical space form. In particular, if $M$ is simply connected, then $M$ is diffeomorphic to the standard n-sphere $\mathbb{S}^{n}$.

2. Preliminaries. Let $M^{n}$ be an $n$-dimensional complete submanifold isometrically immersed into an $(n+p)$-dimensional Riemannian manifold $N^{n+p}$. The following conventions of indices are used throughout.

$$
\begin{gathered}
1 \leq A, B, C, \ldots \leq n+p, \\
1 \leq i, j, k, \ldots \leq n, \\
n+1 \leq \alpha, \beta, \gamma, \ldots \leq n+p .
\end{gathered}
$$

Choose a local orthonormal frame field $\left\{e_{A}\right\}$ in $N^{n+p}$ such that $e_{i}^{\prime}$ s are tangent to $M$. Let $\left\{\omega_{A}\right\}$ be the dual frame field of $\left\{e_{A}\right\}$ and $\left\{\omega_{A B}\right\}$ the connection 1-forms of $N^{n+p}$. Restricting these forms to $M$, we have

$$
\omega_{\alpha i}=\sum_{j} h_{i j}^{\alpha} \omega_{j}, h_{i j}^{\alpha}=h_{j i}^{\alpha} .
$$

The curvature tensor of $N$ and $M$ are denoted by $\bar{R}_{A B C D}$ and $R_{i j k l}$, respectively. The second fundamental form of $M$ is denoted by $h$ and the mean curvature normal field by $\xi$. Set $H=\|\xi\|$ and $c_{\alpha}=\frac{1}{n} \sum_{i} h_{i i}^{\alpha}$. Then we have

$$
\begin{gathered}
h=\sum_{\alpha, i, j} h_{i j}^{\alpha} \omega_{i} \otimes \omega_{j} \otimes e_{\alpha}, \\
\xi=\sum_{\alpha} c_{\alpha} e_{\alpha}, \\
H=\sqrt{\sum_{\alpha} c_{\alpha}^{2}}, \\
R_{i j k l}=\bar{R}_{i j k l}+\sum_{\alpha}\left(h_{i k}^{\alpha} h_{j l}^{\alpha}-h_{i l}^{\alpha} h_{j k}^{\alpha}\right) .
\end{gathered}
$$

The trace free second fundamental form of $M$ is defined by

$$
\stackrel{\circ}{h}=\sum_{i, j, \alpha} \stackrel{\circ}{h}_{i j}^{\alpha} \omega_{i} \otimes \omega_{j} \otimes e_{\alpha}
$$


where $\stackrel{\circ}{h}_{i j}^{\alpha}=h_{i j}^{\alpha}-c_{\alpha} \delta_{i j}$. Let $U M$ denote the unit tangent bundle on $M$ and $U_{x} M$ its fiber over $x \in M$. Then $U M=\bigcup_{x \in M} U_{x} M$, where $U_{x} M=\left\{u \in T_{x} M:\|u\|=1\right\}$.

LEMMA 2.1. Let $M^{n}$ be a submanifold in a Riemannian manifold $N^{n+p}$. Then

$$
\|\stackrel{\circ}{h}(u, v)\| \leq \stackrel{\circ}{h}_{x},
$$

for all unit vectors $u, v \in U_{x} M$ at each point $x \in M$, where $\stackrel{\circ}{h}_{x}:=\max _{w \in U_{x} M}\|\stackrel{\circ}{h}(w, w)\|$.

ProOF. If $u \neq \pm v$, set $y=\frac{u+v}{\|u+v\|}$ and $z=\frac{u-v}{\|u-v\|}$. Then

$$
\begin{aligned}
\stackrel{\circ}{h}(u, v) & =\frac{1}{4}[\stackrel{\circ}{h}(u+v, u+v)-\stackrel{\circ}{h}(u-v, u-v)] \\
& =\frac{1}{4}\left[\|u+v\|^{2} \stackrel{\circ}{h}(y, y)-\|u-v\|^{2} \stackrel{\circ}{h}(z, z)\right] .
\end{aligned}
$$

Using the triangle inequality, we get

$$
\begin{aligned}
\|\stackrel{\circ}{h}(u, v)\| & \leq \frac{1}{4} \stackrel{\circ}{h}_{x}\left(\|u+v\|^{2}+\|u-v\|^{2}\right) \\
& \leq \check{\circ}_{x} .
\end{aligned}
$$

If $u= \pm v$, then $\|\stackrel{\circ}{h}(u, v)\|=\|\stackrel{\circ}{h}(u, u)\| \leq \stackrel{\circ}{h}_{x}$. This completes the proof.

LEMMA 2.2. Let $M^{n}$ be a submanifold in a Riemannian manifold $N^{n+p}$. Then

$$
\left\langle h\left(e_{i}, e_{i}\right), h\left(e_{j}, e_{j}\right)\right\rangle \geq-\stackrel{\circ}{x}_{x}^{2}
$$

for any orthonormal frame field $\left\{e_{i}\right\}$ at each point $x \in M$.

Proof.

$$
\begin{aligned}
\left\|h\left(e_{i}, e_{i}\right)\right\|^{2}+\left\|h\left(e_{j}, e_{j}\right)\right\|^{2}-2\left\langle h\left(e_{i}, e_{i}\right), h\left(e_{j}, e_{j}\right)\right\rangle \\
=\left\|\stackrel{\circ}{h}\left(e_{i}, e_{i}\right)\right\|^{2}+\left\|\stackrel{\circ}{h}\left(e_{j}, e_{j}\right)\right\|^{2}+2\left\langle\stackrel{\circ}{h}\left(e_{i}, e_{i}\right), \xi\right\rangle+2\left\langle\stackrel{\circ}{h}\left(e_{j}, e_{j}\right), \xi\right\rangle+2 H^{2} \\
\quad-2\left(\left\langle h\left(e_{i}, e_{i}\right), \stackrel{\circ}{h}\left(e_{j}, e_{j}\right)\right\rangle+\left\langle\stackrel{\circ}{h}\left(e_{i}, e_{i}\right), \xi\right\rangle+\left\langle\stackrel{\circ}{h}\left(e_{j}, e_{j}\right), \xi\right\rangle+H^{2}\right) \\
=\left\|\stackrel{\circ}{h}\left(e_{i}, e_{i}\right)\right\|^{2}+\left\|\stackrel{\circ}{h}\left(e_{j}, e_{j}\right)\right\|^{2}-2\left\langle\stackrel{\circ}{h}\left(e_{i}, e_{i}\right), \stackrel{\circ}{h}\left(e_{j}, e_{j}\right)\right\rangle \\
\leq 4 \stackrel{\circ}{x}_{x}^{2},
\end{aligned}
$$

where Lemma 2.1 is used in the last inequality. Hence we have

$$
\left\langle h\left(e_{i}, e_{i}\right), h\left(e_{j}, e_{j}\right)\right\rangle \geq \frac{1}{2}\left(\left\|h\left(e_{i}, e_{i}\right)\right\|^{2}+\left\|h\left(e_{j}, e_{j}\right)\right\|^{2}\right)-2 \stackrel{\circ}{h}_{x}^{2} .
$$

Thus,

$$
\begin{aligned}
\left\langle h\left(e_{i}, e_{i}\right), h\left(e_{j}, e_{j}\right)\right\rangle= & \frac{1}{2}\left\langle h\left(e_{i}, e_{i}\right), h\left(e_{j}, e_{j}\right)\right\rangle+\frac{1}{2}\left\langle h\left(e_{i}, e_{i}\right), h\left(e_{j}, e_{j}\right)\right\rangle \\
\geq & \frac{1}{2}\left[\frac{1}{2}\left(\left\|h\left(e_{i}, e_{i}\right)\right\|^{2}+\left\|h\left(e_{j}, e_{j}\right)\right\|^{2}\right)-2 h_{x}^{2}\right] \\
& -\frac{1}{2}\left\|h\left(e_{i}, e_{i}\right)\right\| \cdot\left\|h\left(e_{j}, e_{j}\right)\right\| \\
= & \frac{1}{4}\left[\left\|h\left(e_{i}, e_{i}\right)\right\|^{2}+\left\|h\left(e_{j}, e_{j}\right)\right\|^{2}\right.
\end{aligned}
$$




$$
\begin{aligned}
& \left.-2\left\|h\left(e_{i}, e_{i}\right)\right\| \cdot\left\|h\left(e_{j}, e_{j}\right)\right\|\right]-\stackrel{\circ}{h}_{x}^{2} \\
\geq & -\stackrel{\circ}{h}_{x}^{2} .
\end{aligned}
$$

S. Brendle [5] obtained the following useful result.

Lemma 2.3. Let $\left(M, g_{0}\right)$ be a compact Riemannian manifold of dimension $n \geq 4$. Assume that

$$
R_{1313}+\lambda^{2} R_{1414}+R_{2323}+\lambda^{2} R_{2424}-2 \lambda R_{1234}>0,
$$

for all orthonormal four-frames $\left\{e_{1}, e_{2}, e_{3}, e_{4}\right\}$ and all $\lambda \in[-1,1]$. Then the normalized Ricci flow with initial metric $g_{0}$

$$
\frac{\partial}{\partial t} g(t)=-2 \operatorname{Ric}_{g(t)}+\frac{2}{n} r_{g(t)} g(t),
$$

exists for all time and converges to a positive constant sectional curvature metric as $t \rightarrow \infty$. Here $r_{g(t)}$ denotes the mean value of the scalar curvature of $g(t)$.

Inequality (5) is closely related to the positivity of the isotropic curvature. We refer the readers to [13] for isotropic curvature. As a matter of fact, $R_{1313}+\lambda^{2} R_{1414}+R_{2323}+\lambda^{2} R_{2424}-$ $2 \lambda R_{1234}>0$ holds for all orthonormal four-frames $\left\{e_{1}, e_{2}, e_{3}, e_{4}\right\}$ and all $\lambda \in[-1,1]$ if and only if $M \times \mathbb{R}$ has positive isotropic curvature (see [5, 6]). It follows from Berger's inequality that every manifold with positively pointwise 1/4-pinched sectional curvatures satisfies the curvature condition (5) in Lemma 2.3. In fact, we have the following Berger's inequality [3]

$$
\left|R_{i j k l}\right| \leq \frac{2}{3}\left(K_{\max }-K_{\min }\right),
$$

for all distinct indices $i, j, k, l$. Here $K_{\max }$ and $K_{\min }$ are the maximum and minimum of the sectional curvatures at a point of $M$. The curvature assumption implies that $K_{\max }<4 K_{\min }$. So we have

$$
\begin{aligned}
R_{1313} & +\lambda^{2} R_{1414}+R_{2323}+\lambda^{2} R_{2424}-2 \lambda R_{1234} \\
& \geq 2\left(1+\lambda^{2}\right) K_{\min }-\frac{4}{3}|\lambda|\left(K_{\max }-K_{\min }\right) \\
& \geq 2\left(1+\lambda^{2}\right) K_{\min }-4|\lambda| K_{\min } \\
& =2(1-|\lambda|)^{2} K_{\min } \\
& \geq 0 .
\end{aligned}
$$

Observe that at least one of the second and last inequalities is strict, which implies $R_{1313}+$ $\lambda^{2} R_{1414}+R_{2323}+\lambda^{2} R_{2424}-2 \lambda R_{1234}>0$. In fact, the 1/4-pinched differentiable sphere theorem is an immediate consequence of Lemma 2.3.

We also need the following topological lemma due to H. B. Lawson and J. Simons [11]. 
LEMMA 2.4. Let $M^{n}$ be a compact submanifold in a unit sphere $\mathbb{S}^{n+p}$. Let $k$ and $l$ be positive integers with $k+l=n$. If the following inequality

$$
\sum_{j=k+1}^{n} \sum_{i=1}^{k}\left(2\left\|h\left(e_{i}, e_{j}\right)\right\|^{2}-\left\langle h\left(e_{i}, e_{i}\right), h\left(e_{j}, e_{j}\right)\right\rangle\right)<k \cdot l,
$$

holds for any orthonormal basis $\left\{e_{1}, e_{2}, \ldots, e_{n}\right\}$ of tangent space $T_{x} M$ at any point $x \in M$, then there does not exist any stable $k$-current, and

$$
H_{k}(M, \mathbb{Z})=H_{l}(M, \mathbb{Z})=0,
$$

where $H_{i}(M, \mathbb{Z})$ is the $i$-th homology group of $M$ with integer coefficients.

3. Proof of theorems. We first give the proof of Theorem 1.3.

Proof of Theorem 1.3. Since $K_{M}>\frac{(n-2) c}{n}+\frac{n H^{2}}{8}>0$, we obtain that $M$ is a compact submanifold by Myers' Theorem.

Suppose that $\left\{e_{1}, e_{2}, e_{3}, e_{4}\right\}$ is an orthonormal four-frame and $\lambda \in \mathbb{R}$. We extend $\left\{e_{1}, e_{2}, e_{3}, e_{4}\right\}$ to an orthonormal frame $\left\{e_{1}, \ldots, e_{n}\right\}$. From the Gauss equation (2) we get

$$
R_{i j i j}=c+\sum_{\alpha} h_{i i}^{\alpha} h_{j j}^{\alpha}-\sum_{\alpha}\left(h_{i j}^{\alpha}\right)^{2} .
$$

From the assumption we obtain

$$
\sum_{\alpha} h_{i i}^{\alpha} h_{j j}^{\alpha}-\sum_{\alpha}\left(h_{i j}^{\alpha}\right)^{2}>\frac{n H^{2}}{8}-\frac{2 c}{n} .
$$

This together with (1) implies

$$
\begin{aligned}
R_{1313}+ & \lambda^{2} R_{1414}+R_{2323}+\lambda^{2} R_{2424}-2 \lambda R_{1234} \\
> & \left(1+\lambda^{2}\right)\left(\frac{2(n-2)}{n} c+\frac{n H^{2}}{4}\right)-2 \lambda \sum_{\alpha}\left(h_{13}^{\alpha} h_{24}^{\alpha}-h_{14}^{\alpha} h_{23}^{\alpha}\right) \\
\geq & \left(1+\lambda^{2}\right)\left(\frac{2(n-2)}{n} c+\frac{n H^{2}}{4}\right)-|\lambda| \sum_{\alpha} \sum_{i=3}^{n}\left[\left(h_{1 i}^{\alpha}\right)^{2}+\left(h_{2 i}^{\alpha}\right)^{2}\right] \\
\geq & \left(1+\lambda^{2}\right)\left(\frac{2(n-2)}{n} c+\frac{n H^{2}}{4}\right)-|\lambda|\left[\sum_{\alpha}\left(h_{11}^{\alpha}+h_{22}^{\alpha}\right)\left(\sum_{i=3}^{n} h_{i i}^{\alpha}\right)\right. \\
& \left.-\left(\frac{n(n-2) H^{2}}{4}-\frac{4(n-2) c}{n}\right)\right] \\
\geq & \left(1+\lambda^{2}\right)\left(\frac{2(n-2)}{n} c+\frac{n H^{2}}{4}\right)-|\lambda| \frac{n^{2}}{4} H^{2}+|\lambda|\left(\frac{n(n-2) H^{2}}{4}-\frac{4(n-2) c}{n}\right) \\
= & \frac{2(n-2)}{n}(1-|\lambda|)^{2} c+\frac{n}{4}(1-|\lambda|)^{2} H^{2} \\
\geq & 0 .
\end{aligned}
$$


Hence, the inequality (5) holds for all $\lambda \in[-1,1]$, which implies that $M$ is diffeomorphic to a spherical space form by Lemma 2.3. On the other hand, since $K_{M}>\frac{(n-2) c}{n}+\frac{n H^{2}}{8} \geq$ $\frac{c}{2}+\frac{n H^{2}}{8}$, from Theorem 1.1 we know that $M$ is homeomorphic to $\mathbb{S}^{n}$. Combining the results above, we get that $M$ is diffeomorphic to $\mathbb{S}^{n}$.

In the following we consider the submanifolds with restriction on $\stackrel{\circ}{\text {. }}$

LEMMA 3.1. Let $M$ be an $n(\geq 4)$-dimensional submanifold in an $(n+p)$-dimensional point-wise $\delta(>1 / 4)$-pinched Riemannian manifold $N^{n+p}$. If

$$
\|\stackrel{\circ}{h}(u, u)\|^{2}(x)<\frac{4}{9} \bar{K}_{\max }(x)\left(\delta-\frac{1}{4}\right), \quad \text { for all } u \in U_{x} M, \quad x \in M,
$$

where $\inf _{x \in N} \bar{K}_{\max }(x)>0$, then inequality (5) is satisfied for all orthonormal four-frames $\left\{e_{1}, e_{2}, e_{3}, e_{4}\right\}$ and $\lambda \in[-1,1]$.

ProOF. For any point $x \in M$, let $\stackrel{\circ}{h}_{x}:=\max _{w \in U_{x} M}\|\stackrel{\circ}{h}(w, w)\|$. We suppose $\left\{e_{1}, e_{2}, e_{3}, e_{4}\right\}$ to be an orthonormal four-frame and $\lambda \in[-1,1]$. By the Gauss equation we have

$$
\begin{aligned}
R_{1313}+ & \lambda^{2} R_{1414}+R_{2323}+\lambda^{2} R_{2424}-2 \lambda R_{1234} \\
= & \bar{R}_{1313}+\left\langle h\left(e_{1}, e_{1}\right), h\left(e_{3}, e_{3}\right)\right\rangle-\left\|h\left(e_{1}, e_{3}\right)\right\|^{2} \\
& +\lambda^{2}\left[\bar{R}_{1414}+\left\langle h\left(e_{1}, e_{1}\right), h\left(e_{4}, e_{4}\right)\right\rangle-\left\|h\left(e_{1}, e_{4}\right)\right\|^{2}\right] \\
& +\left[\bar{R}_{2323}+\left\langle h\left(e_{2}, e_{2}\right), h\left(e_{3}, e_{3}\right)\right\rangle-\left\|h\left(e_{2}, e_{3}\right)\right\|^{2}\right] \\
& +\lambda^{2}\left[\bar{R}_{2424}+\left\langle h\left(e_{2}, e_{2}\right), h\left(e_{4}, e_{4}\right)\right\rangle-\left\|h\left(e_{1}, e_{4}\right)\right\|^{2}\right] \\
& -2|\lambda|\left[\bar{R}_{1234}+\left\langle h\left(e_{1}, e_{3}\right), h\left(e_{2}, e_{4}\right)\right\rangle-\left\langle h\left(e_{1}, e_{4}\right), h\left(e_{2}, e_{3}\right)\right\rangle\right] .
\end{aligned}
$$

Applying Lemma 2.1, Lemma 2.2 and $h\left(e_{i}, e_{j}\right)=\stackrel{\circ}{h}\left(e_{i}, e_{j}\right), i \neq j$, we obtain

$$
\begin{aligned}
R_{1313}+ & \lambda^{2} R_{1414}+R_{2323}+\lambda^{2} R_{2424}-2 \lambda R_{1234} \\
\geq & \bar{R}_{1313}-2 h_{x}^{2}+\lambda^{2}\left[\bar{R}_{1414}-2 h_{x}^{2}\right] \\
& +\bar{R}_{2323}-2 h_{x}^{2}+\lambda^{2}\left[\bar{R}_{2424}-2 h_{x}^{2}\right] \\
& -2|\lambda|\left[\bar{R}_{1234}+2 h_{x}^{2}\right] .
\end{aligned}
$$

Since we have Berger's inequality $\left|\bar{R}_{1234}\right| \leq \frac{2}{3}\left(\bar{K}_{\max }-\bar{K}_{\min }\right)$, the assumption (6) implies that

$$
\begin{aligned}
R_{1313} & +\lambda^{2} R_{1414}+R_{2323}+\lambda^{2} R_{2424}-2 \lambda R_{1234} \\
& >\left(2+2 \lambda^{2}\right)\left[\bar{K}_{\min }-\frac{8}{9} \bar{K}_{\max }\left(\delta-\frac{1}{4}\right)\right]-2|\lambda|\left[\frac{2}{3}\left(\bar{K}_{\max }-\bar{K}_{\min }\right)+\frac{8}{9} \bar{K}_{\max }\left(\delta-\frac{1}{4}\right)\right] \\
& \geq\left(2+2 \lambda^{2}\right)\left[\delta \bar{K}_{\max }-\frac{8}{9} \bar{K}_{\max }\left(\delta-\frac{1}{4}\right)\right]-2|\lambda|\left[\frac{2}{3}(1-\delta) \bar{K}_{\max }+\frac{8}{9} \bar{K}_{\max }\left(\delta-\frac{1}{4}\right)\right] \\
& =\frac{2}{9}\left(1+\lambda^{2}-2|\lambda|\right)(\delta+2) \bar{K}_{\max } \\
\geq & 0 .
\end{aligned}
$$


This completes the proof of the Lemma 3.1.

Now we give the proof of Theorem 1.5.

Proof of Theorem 1.5. Since $\|h(u, u)\|^{2}(x)<\frac{4}{9}\left(\delta-\frac{1}{4}\right) \bar{K}_{\max }$ for any $u \in U_{x} M$, from the Gauss equation, we know that the sectional curvature of $M$ is bounded from bellow by $\frac{2+\delta}{9} \bar{K}_{\max } \geq \frac{2+\delta}{9} \inf _{x \in M} \bar{K}_{\max }(x)>0$. By Myers' Theorem, $M$ is a compact submanifold.

When $n=2$, it's easy to see that $M$ is diffeomorphic to $\mathbb{S}^{2}$ or $\mathbb{R P}^{2}$. When $n=3$, Hamilton's Theorem [9] says that $M$ is diffeomorphic to a spherical space form. When $n \geq 4$, by Lemma 2.3 and Lemma 3.1, we see that $M$ is diffeomorphic to a spherical space form. In particular, if $M$ is simply connected, then $M$ must be diffeomorphic to the standard unit $n$-sphere $\mathbb{S}^{n}$. This completes the proof of Theorem 1.5.

LEMMA 3.2. Let $M^{n}$ be an $n$-dimensional complete submanifold in the unit sphere $\mathbb{S}^{n+p}$. If

$$
\|\stackrel{\circ}{h}(u, u)\|^{2}<\frac{1}{3}, \quad \text { for all } u \in U M
$$

then $M$ is simply connected.

PROOF. It is easy to see that the sectional curvature of $M$ is bounded by $\frac{1}{3}$ from below. Hence $M$ is compact by Myers' Theorem.

Let $\left\{e_{1}, e_{2}, \ldots, e_{n}\right\}$ be any orthonormal basis of the tangent space at any point $x \in M$. Using Lemma 2.1 and Lemma 2.2, we have

$$
\begin{gathered}
\left\|h\left(e_{i}, e_{j}\right)\right\|=\left\|\stackrel{\circ}{h}\left(e_{i}, e_{j}\right)\right\|<\frac{1}{3}, \\
\left\langle h\left(e_{i}, e_{i}\right), h\left(e_{j}, e_{j}\right)\right\rangle>-\frac{1}{3},
\end{gathered}
$$

for any unit vector field $e_{i}$ and $e_{j}(i \neq j)$. Hence for any $1 \leq k \leq n-1$,

$$
\begin{aligned}
\sum_{j=k+1}^{n} & \sum_{i=1}^{k}\left(2\left\|h\left(e_{i}, e_{j}\right)\right\|^{2}-\left\langle h\left(e_{i}, e_{i}\right), h\left(e_{j}, e_{j}\right)\right\rangle\right) \\
& <\sum_{j=k+1}^{n} \sum_{i=1}\left(\frac{2}{3}+\frac{1}{3}\right) \\
& =k l .
\end{aligned}
$$

By Lemma 2.4, there does not exist any stable integral current in $M$.

Suppose that $\pi_{1}(M) \neq 0$. Since $M$ is compact, it follows from a classical theorem due to Cartan and Hadamard (see [4]) that there exists a minimal closed geodesic in any nontrivial homotopy class in $\pi_{1}(M)$. Then we get a contradiction. Therefore, $\pi_{1}(M)=0$ and $M$ is simply connected.

Theorem 1.4 is an immediate consequence of Lemma 3.2 and Theorem 1.5.

Acknowledgements. The authors would like to thank the referee for his/her valuable suggestions. 


\section{REFERENCES}

[1] M. Anderson, Convergence and rigidity of manifolds under Ricci curvature bounds, Invent. Math. 102 (1990), 429-445.

[2] M. Berger, Les variétés Riemanniennes (1/4)-pincées, Ann. Scuola Norm. Sup. Pisa 14 (1960), 161-170.

[3] M. Berger, Sur quelques variétés riemanniennes suffisamment pincées, Bull. Soc. Math. France 88 (1960), 51-71.

[ 4 ] R. BotT, Lectures on Morse Theory, old or new, Bull. Amer. Math. Soc. 7 (1982), 331-358.

[ 5 ] S. BREndLE, A general convergence result for the Ricci flow in higher dimensions, Duke Math. J. 145 (2008), 585-601.

[6] S. Brendle And R. Schoen, Manifolds with 1/4-pinched curvature are space forms, J. Amer. Math. Soc. 22 (2009), 287-307.

[ 7 ] H. Gauchman, Minimal submanifolds of a sphere with bounded second fundamental form, Tran. Amer. Math. Soc. 292 (1986), 779-791.

[ 8 ] K. Grove And K. Shiohama, A generalized sphere theorem, Ann. of Math. 106 (1977), 201-211.

[9] R. Hamilton, Three-manifolds with positive Ricci curvature, J. Differential Geom. 17 (1982), 255-306.

[10] W. Klingenberg, Über Riemannsche Mannigfaltigkeiten mit positiver Krümmung, Comment. Math. Helv. 35 (1961), 47-54.

[11] H. B. LAwSON AND J. Simons, On stable currents and their application to global problems in real and complex geometry, Ann. of Math. 98 (1973), 427-450.

[12] P. F. LEUNG, On the topology of a compact submanifold of a sphere with bounded second fundamental form, Manus. Math. 79 (1993), 183-185.

[13] M. Micallef And J. D. Moore, Minimal two-spheres and the topology of manifolds with positive curvature on totally isotropic two-planes, Ann. of Math. 127 (1988), 199-227.

[14] Y. Otsu, K. Shiohama And T. Yamaguchi, A new version of differentiable sphere theorem, Invent. Math. 98 (1989), 219-228.

[15] G. Perelman, Manifolds of positive Ricci curvature with almost maximal volume, J. Amer. Math. Soc. 7 (1994), 299-305.

[16] H. E. RAUCH, A contribution to differential geometry in the large, Ann. of Math. 54 (1951), 38-55.

[17] K. Shiонамa, Sphere theorems, Handbook of Differential Geometry, Vol. 1, F.J.E. Dillen and L.C.A. Verstraelen (eds.), Elsevier Science B.V., Amsterdam, 2000.

[18] K. Shiohama And H. W. XU, The topological sphere theorem for complete submanifolds, Compositio Math. 107 (1997), 221-232.

[19] H. W. XU AND W. FANG, Geometric and topological rigidity of complete submanifolds, preprint, 2001.

[20] H. W. XU AND J. R. GU, An optimal differentiable sphere theorem for complete manifolds, Math. Res. Lett. 17 (2010), 1111-1124.

[21] H. W. XU, W. FAng And F. XIAng, A generalization of Gauchman's rigidity theorem, Pacific J. Math. 228 (2006), 185-199.

[22] H. W. XU, F. HuAng AND F. Xiang, An extrinsic rigidity theorem for submanifolds with parallel mean curvature in a sphere, Kodai Math. J. 34 (2011), 85-104.

[23] H. W. XU AND G. X. YANG, Topological sphere theorems for submanifolds with positive curvature, preprint, 2008.

[24] H. W. XU AND E. T. ZhaO, Topological and differentiable sphere theorems for complete submanifolds, Comm. Anal. Geom. 17 (2009), 565-585.

[25] T. YAmAGUCHI, Lipschitz convergence of manifolds of positive Ricci curvature with large volume, Math. Ann. 284 (1989), 423-436. 
Center of Mathematical Sciences

ZHEJIANG UNIVERSITY

HANGZHOU 310027

P. R. CHINA

E-mail addresses: xuhw@cms.zju.edu.cn, huangfei@cms.zju.edu.cn

zhaoet@cms.zju.edu.cn 